\title{
Research on The Relationship Between Bearing Preload and System Natural Frequency
}

\author{
Wang Yan-shuang, Li Shao-chuan \\ College of Mechanical Engineering, Tianjin University of Technology and Education, Tianjin 300222, \\ China \\ hkd_wang_yan_shuang@126.com
}

\begin{abstract}
Keywords: bearing; preload; natural frequency; stiffness; span
Abstract. A special-purpose testing device for bearing preload was developed. The relationship between preload and system natural frequency was researched with the test rig. The test data of bearing preload changing with system natural frequency was fitted. The results show that the preload test rig has reflected the bearing pre-tight characteristic to some extent. The system natural frequency increases with the increasing bearing preload. In case of the same bearing preload, the system natural frequency increases with the increase in the bearing span. The precision of the fitted relationship formula of preload-natural frequency-bearing span is higher.
\end{abstract}

\section{Introduction}

By a long period of experiment research, a lot of experiences have been accumulated in determining the bearing preload, some effective methods have also been found to determine the optimum preload ${ }^{[1,2]}$. However, these methods can be obtained either from a theoretical determination or actually measurement of the preload, which has great labor intensity and is easily influenced by subjective factors, the accuracy and efficiency ${ }^{[3-5]}$. For special occasions of high precision or limited operating space, the traditional method can not meet the requirement ${ }^{[6]}$. Therefore, in order to obtain the actual bearing preload, a new method to determine the preload must be found. The paper discards the traditional preload testing concept, studies the relationship between the bearing preload and the natural frequency of the system, and determines the actual bearing preload according to the natural frequency. The method can not only improve the testing accuracy and efficiency, but also greatly reduces the labor intensity.

\section{Test principle}

The research theory of this paper comes from the relationship between the bearing preload and the support stiffness, and the relationship between the stiffness and the natural frequency. Because the bearing stiffness can be changed with preload, and the bearing stiffness impacts on the system natural frequency, the establishment of relationship between preload, bearing stiffness and natural frequency can be carried out. In this paper, taking an angular contact ball bearing for example, a testing system was established, which can study the relationship between preload and system natural frequency as well as the influence of bearing support span on system natural frequency.

Angular contact ball bearing is used in the test, the corresponding parameters are as following:

Rolling element diameter $\mathrm{D}_{\mathrm{w}}: 9.525 \mathrm{~mm}$

Number of rolling elements Z: 12

Contact angle $\alpha: 25^{\circ}$

\section{Testing system}

The testing system is made up of bearing support system, loading equipment, data acquisition and analysis system, mathematical software analysis system. Angular contact ball bearing 7206AC is preloaded by loading equipment. By means of force sensor system, data is collected and the monitoring results are showed. The test results between preload and system natural frequency can be obtained by knocking with LC02A force hammer, picking array with accelerating sensor, data 
sampling and analyzing with YE6261B dynamic data testing and collecting system. The testing sketch is shown in Fig 1.

The bearing support system is the important part of the test system, which makes up the typical preload testing equipment together with the loading system to load and output signals. Data collecting system is made up of sensor which can transmit data to the computer. Computer data processing and result display system is used to process the received signal and show the results directly. The last work is data analyzing and curve fitting.

The design of the testing equipment is based on the bearing location in engineering and the actual bearing working condition. In general, angular contact bearing has two installing methods, one of them is face to face installation, the other is back to back installation. The installation of a pair

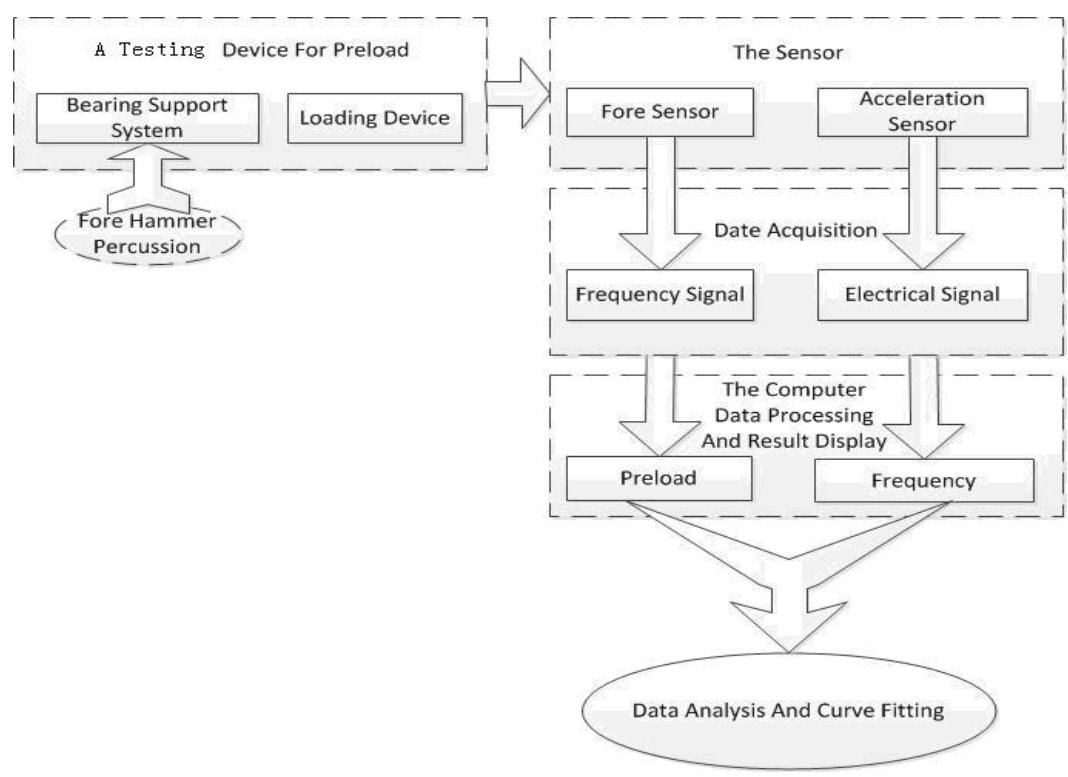

Fig.1 Diagram of test system

of bearings in the testing system is face to face installation, which is shown in Fig 2. The testing system includes table base 1, working table 2, the test shaft 3 and loading and testing components etc. The external force is applied on the outer ring of the bearing 8 on the right through the gland 7, it can be transmitted to the inner ring of the bearing 8 on the left by the rolling element,the inner ring of the bearing on the right and the test shaft 3 . Finally, the force can be transmitted to the housing 9 by the rolling element, the inner ring, outer ring of the bearing on the left, gland 10 and screw 11. The loading equipment 6 is fixed on the side of the bearing.

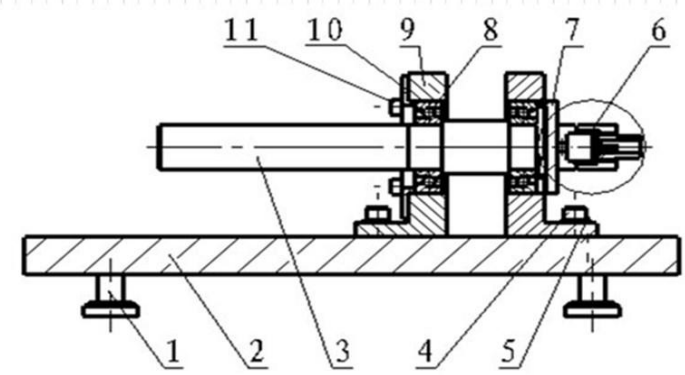
1. Table Base 2. Working Table 3. Test Shaft 4. Screw
5. Located Pin 6. Loading Device 7. Gland 8. Bearing
9. Bearing Housing 10. Gland (2) 11. Screw

Fig.2 Test rig

\section{Experiment}

Determination of actual bearing preload is an assurance of support system precision of the bearings which is used in high precision, or special occasion. With the same support span, the relationship 
between bearing preload and system natural frequency can be indicated by the monitoring in the test, which can be the basis of new theory.

In the paper, testing system of preload and natural frequency makes use of bearing preload testing equipment to investigate. The test include 3 spans, which are respectively $95 \mathrm{~mm}, 75 \mathrm{~mm}$ and $55 \mathrm{~mm}$. The test data and analysis are respectively preload $(\mathrm{N})$ and natural frequency $(\mathrm{Hz})$. At last the results of relationship between preload and natural frequency has been obtained. Considered with support span, by means of professional software, the functional relationship between preload, natural frequency and support span can be discovered.

\section{Test results and analysis}

According to the structural characteristics of the test device, testing can be divided into three groups A, B, C, the bearing span were $95 \mathrm{~mm}, 75 \mathrm{~mm}, 55 \mathrm{~mm}$. Besides support span, other parameters approximately remained the same.

Natural frequency of the test. Collected response signal by hammering, shown in Figure 3:

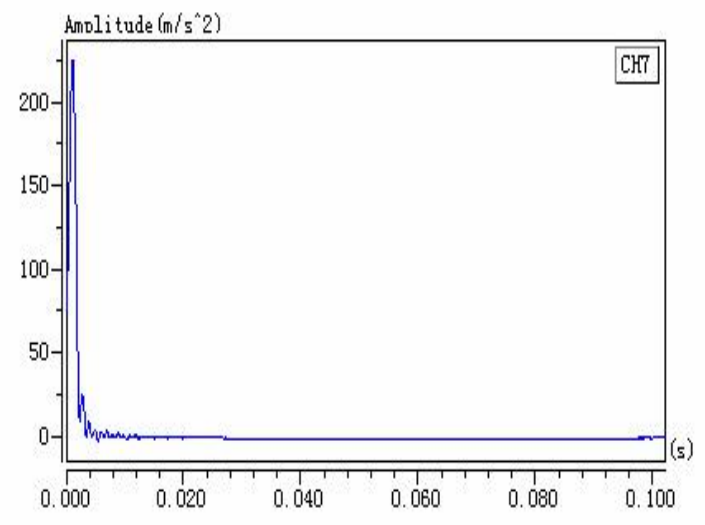

a) Force signal

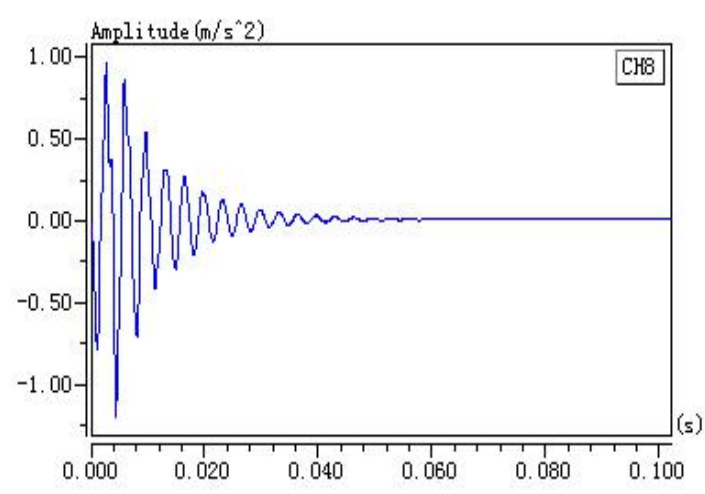

b) Response signal

Fig.3 Test signal

Statistics and fitting analysis. In the experiment, the test system can acquire the relation curve of bearing preload versus the natural frequency under 3 spans, as shown in Figure 4.

The regression analysis can be carried out with Maikuate method. Then, the relationship between preload, natural frequency and span can be achieved as following:

$$
F=p_{1}+p_{2} \times f_{n}+p_{3} \times f_{n}^{2}+p_{4} \times f_{n}^{3}+p_{5} \times f_{n}^{4}+p_{6} \times L+p_{7} \times L^{2} \text {. }
$$

where:

$$
\begin{aligned}
& p_{1}=183.365297851066 \\
& p_{2}=-1.88841146172325 \\
& p_{3}=1.74692100048334 \mathrm{E}-02 \\
& p_{4}=-5.79198372685408 \mathrm{E}-05 \\
& p_{5}=6.6339756332295 \mathrm{E}-08 \\
& p_{6}=-2.92122384550087 \\
& p_{7}=1.39718208402563 \mathrm{E}-02
\end{aligned}
$$

The formula reflects the relationship between preload, natural frequency and span. The total correlation coefficient $\mathrm{R}$ is 0.99084 . The relationship between them are shown in Figure 5.

Results comparison. Table 1, Table 2 and Table 3 display respectively the comparison results of the calculated values with the experimental data under different support span $95 \mathrm{~mm}, 75 \mathrm{~mm}$ and $55 \mathrm{~mm}$. 


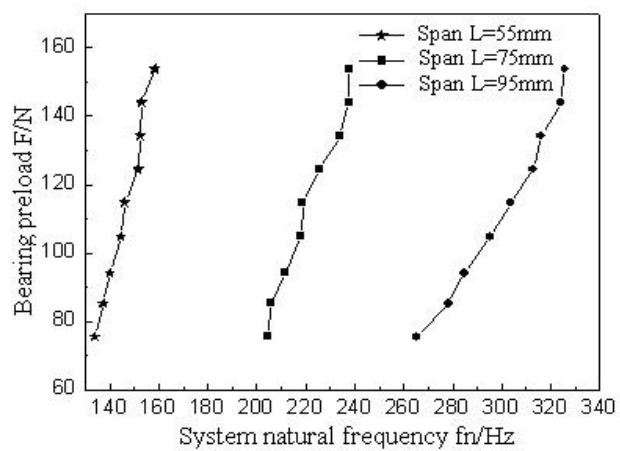

Fig.4 Fitted curve of bearing preload-system natural frequency

From the experiment it can be seen that at the same support span, natural frequency increases with the increase in bearing preload, at the same preload, natural frequency increases with the increasing support span.

Table 1 Calculated value and experimental value comparison (support span is $95 \mathrm{~mm}$ )

\begin{tabular}{cccc}
\hline Frequency $(\mathrm{Hz})$ & Experiment Value $(\mathrm{N})$ & Calculated value $(\mathrm{N})$ & Relative Error $(\%)$ \\
\hline 320.22 & 74.36 & 75.5 & 1.5 \\
326.803 & 87.57 & 85.3 & 2.7 \\
332.208 & 93.49 & 94.1 & 0.6 \\
338.269 & 104.14 & 104.9 & 0.7 \\
343.343 & 113.92 & 114.7 & 0.7 \\
348.068 & 126.90 & 124.5 & 1.9 \\
352.492 & 132.55 & 134.3 & 1.3 \\
356.655 & 147.77 & 144.1 & 2.5 \\
360.588 & 150.60 & 153.9 & 2.1 \\
\hline
\end{tabular}

Table 2 Calculated value and experimental value comparison (support span is $75 \mathrm{~mm}$ )

\begin{tabular}{cccc}
\hline Frequency $(\mathrm{Hz})$ & Experiment Value $(\mathrm{N})$ & Calculated value $(\mathrm{N})$ & Relative Error $(\%)$ \\
\hline 236.361 & 77.58 & 75.5 & 2.8 \\
241.219 & 80.86 & 85.3 & 5.2 \\
245.209 & 95.06 & 94.1 & 1 \\
249.683 & 109.67 & 104.9 & 4.5 \\
253.428 & 111.06 & 114.7 & 3.2 \\
256.915 & 125.04 & 124.5 & 0.4 \\
260.181 & 139.72 & 134.3 & 4.0 \\
263.254 & 146.15 & 144.1 & 1.4 \\
266.157 & 146.16 & 153.9 & 5.0 \\
\hline
\end{tabular}

It can be seen from Table 1 that the maximum error between calculated value and experimental value is approximately $2.7 \%$ and the minimum error is $0.6 \%$. From Table 2 it can be seen that the maximum error betweeen calculated value and experimental value is approximately $5.2 \%$ and the minimum error is $0.4 \%$. From Table 3 it can be seen that the maximum error betweeen calculated 
value and experimental value is approximately $5.6 \%$ and the minimum error is $0.004 \%$.

Table 3 Calculated value and experimental value comparison (support span is 55mm)

\begin{tabular}{cccc}
\hline Frequency $(\mathrm{Hz})$ & Experiment Value $(\mathrm{N})$ & Calculated value $(\mathrm{N})$ & $\begin{array}{c}\text { Relative Error } \\
(0<1\end{array}$ \\
\hline 155.025 & 75.06 & 75.5 & 0.6 \\
158.212 & 85.30 & 85.3 & 0.004 \\
160.829 & 93.39 & 94.1 & 0.75 \\
163.763 & 107.49 & 104.9 & 2.5 \\
166.220 & 113.57 & 114.7 & 0.99 \\
168.507 & 131.40 & 124.5 & 5.5 \\
170.649 & 134.50 & 134.3 & 0.15 \\
172.664 & 136.05 & 144.1 & 5.6 \\
174.568 & 154.54 & 153.9 & 0.4 \\
\hline
\end{tabular}

\section{Conclusions}

A test equipment of bearing preload - the natural frequency has been indigenously developed. Test performance is stable and test data are reliable. A new test method was presented for actual bearing preload measurement, namely making use of the natural frequency of the test system to investigate the bearing preload. The natural frequency increases with the increasing bearing preload at the same bearing support system. At the same preload, the natural frequency of the system increases with the increasing bearing span. Bearing preload magnitude can be determined with the fitting results between preload-natural frequency-bearing span relation. The error between test preload value and the calculated value is less than $5.6 \%$.

\section{Acknowledgements}

This work was financially supported by the national natural science foundation of China (51475143), the national natural science foundation of China (51105131) and Tianjin natural science foundation (16JCYBJC18900).

\section{References}

[1] Shan Xiao-biao, Xie Tao, Chen Wei-shan. Novel Approach for Determining the Optimal Axial Preload of Simulating a Rotary Table Spindle System [J]. Journal of Zhejiang University Scien A. 2007, 8 (5): 812-817.

[2] Wang Fucheng. A Method of Determining the Bearing Preload [J]. Modern Components, 2006(4): $80-81$.

[3] Li Hong-guang. The Significance of the Rolling Bearing Preload and the Estimation of Pretightening and Adjust [J]. Journal of Mechanical Engineering, 2004, 42 (481): 45-48.

[4] Li Weimin. Pre-tightness Stiffness Calculation of Axial Positional Tapered Roller Bearing[J]. Journal of Bearing, 2004, (5): 1-3.

[5] Mechanical Design Manual Board. Mechanical Design Manual [M]. Beijing: Mechanical Industry Press, 2007. (26), 11-126.

[6] Jia Qunyi, Deng Si-er. Design Principle of Rolling Bearing [M]. Luoyang: Bearing Research Institute of Henan University of Science and Technology, 2005.89-106. 